\title{
High neutrophil-to-lymphocyte ratio following stereotactic body radiation therapy is associated with poor clinical outcomes in patients with borderline resectable and locally advanced pancreatic cancer
}

\author{
Abhinav V. Reddy ${ }^{1}$, Colin S. Hill ${ }^{1}$, Shuchi Sehgal ${ }^{1}$, Jin $\mathrm{He}^{2}$, Lei Zheng ${ }^{3}$, Joseph M. Herman ${ }^{4}$, \\ Jeffrey Meyer ${ }^{1}$, Amol K. Narang ${ }^{1}$ \\ ${ }^{1}$ Department of Radiation Oncology \& Molecular Radiation Sciences, Johns Hopkins University School of Medicine, Sidney Kimmel Cancer Center, \\ Baltimore, MD, USA; ${ }^{2}$ Department of Surgery, Johns Hopkins University School of Medicine, Sidney Kimmel Cancer Center, Baltimore, MD, \\ USA; ${ }^{3}$ Department of Oncology, Johns Hopkins University School of Medicine, Sidney Kimmel Cancer Center, Baltimore, MD, USA; ${ }^{4}$ Department \\ of Radiation Oncology, Northwell Health, New Hyde Park, NY, USA \\ Contributions: (I) Conception and design: AK Narang, AV Reddy; (II) Administrative support: None; (III) Provision of study materials or patients: \\ S Sehgal, AV Reddy; (IV) Collection and assembly of data: S Sehgal, AV Reddy, CS Hill; (V) Data analysis and interpretation: AV Reddy; (VI) \\ Manuscript writing: All authors; (VII) Final approval of manuscript: All authors. \\ Correspondence to: Abhinav V. Reddy, MD. 401 N Broadway, Baltimore, MD 21231, USA. Email: aredd1990@gmail.com.
}

Background: The purpose of this study is to report on the prognostic role of pre- and post-stereotactic body radiation therapy (SBRT) neutrophil-to-lymphocyte ratio (NLR) in a cohort of patients with borderline resectable (BRPC) and locally advanced pancreatic cancer (LAPC) who was treated with multiagent induction chemotherapy followed by five-fraction SBRT.

Methods: Patients treated with multi-agent induction chemotherapy followed by SBRT from August 2016 to January 2019 and who had laboratory values available for review were included in the study. Univariate (UVA) and multivariate analyses (MVA) were performed to determine associations between pre-/postSBRT NLR and overall survival (OS), local progression-free survival (LPFS), distant metastasis-free survival (DMFS), and progression-free survival (PFS).

Results: A total of 156 patients were treated with multi-agent induction chemotherapy followed by SBRT and had laboratory values available for review. On UVA, chemotherapy duration $\geq 4$ months, poorly differentiated disease, inability to undergo resection, pre-SBRT ANC $\geq 3.7$ No./ $\mu \mathrm{L}$, pre-SBRT NLR $\geq 2.3$, and post-SBRT NLR $\geq 2.6$ were associated with worse OS. Patients with post-SBRT NLR $\geq 2.6$ had a median OS of 16.7 months versus median OS not yet reached in patients with post-SBRT <2.6 $(\mathrm{P}=0.009)$. On MVA, poorly differentiated disease [hazard ratio $(\mathrm{HR})=1.82,95 \% \mathrm{CI}: 1.04-3.18, \mathrm{P}=0.035$ ], inability to undergo resection ( $\mathrm{HR}=2.17,95 \% \mathrm{CI}$ : $1.25-3.70, \mathrm{P}=0.006$ ), and post-SBRT NLR $\geq 2.6$ (HR =2.55, 95\% CI: 1.20-5.45, $\mathrm{P}=0.015)$ were associated with inferior OS. On UVA, baseline CA 19-9 $\geq 219 \mathrm{U} / \mathrm{mL}$, pre-SBRT platelet count $\geq 157 \times 1,000 / \mu \mathrm{L}$, and post-SBRT NLR $\geq 2.6$ were associated with inferior LPFS. Patients with post-SBRT NLR $\geq 2.6$ had a median LPFS of 18.3 months versus median LPFS not yet reached in patients with post-SBRT $<2.6(\mathrm{P}=0.028)$. On MVA, only post-SBRT NLR $\geq 2.6$ was associated with worse LPFS (HR $=3.22$, 95\% CI: $1.04-9.98, \mathrm{P}=0.043)$.

Conclusions: Post-SBRT NLR $\geq 2.6$ predicted for inferior OS and LPFS in BRPC/LAPC patients treated with multi-agent chemotherapy and SBRT. These findings highlight the importance of further elucidating the immunologic effects of radiation therapy in this setting, which may have significant implications on both radiation design as well as combination strategies.

$\wedge$ ORCID: 0000-0001-9209-2923.

(C) Journal of Gastrointestinal Oncology. All rights reserved. 
Keywords: Stereotactic body radiation therapy (SBRT); neutrophil-to-lymphocyte ratio (NLR); localized pancreatic cancer; overall survival (OS)

Submitted Aug 20, 2021. Accepted for publication Nov 24, 2021.

doi: 10.21037/jgo-21-513

View this article at: https://dx.doi.org/10.21037/jgo-21-513

\section{Introduction}

Pancreatic cancer is the third most common cause of cancer related deaths in the US, accounting for over 48,000 deaths each year (1). Treatment usually involves a combination of chemotherapy, radiation therapy, and surgical resection (2). Even with aggressive therapy for patients with localized disease at presentation, prognosis remains guarded, with 5 -year overall survival (OS) of less than $15 \%$, for example, among patients with borderline resectable (BRPC) and/or locally advanced pancreatic cancer (LAPC) $(1,3)$.

The role of radiation therapy for localized pancreatic cancer remains controversial. Currently, radiation therapy is administered for the purpose of margin sterilization and local recurrence risk reduction in the neoadjuvant setting and for improving local progression-free survival (LPFS) and preventing morbidity from disease progression in the unresectable setting (4-13). Two randomized studies have shown that neoadjuvant chemoradiation is associated with higher rates of $\mathrm{R} 0$ resection and improved OS in BRPC $(14,15)$. However, the recent Alliance A021510 trial did not show a benefit of pre-operative chemoradiation versus pre-operative chemotherapy for patients with BRPC (16). Given such conflicting results, a more complete mechanistic understanding of the impact of radiation therapy, beyond classical radiobiologic DNA damage pathways, is critical to optimizing the manner in which radiation is delivered for this patient population. As an example, conformal, hypofractionation is often cited as a potential tool to promote antigen release and evoke pro-immunogenic pathways, but supporting data is lacking.

Inflammation has been associated with chronic diseases including diabetes, heart failure, chronic obstructive pulmonary disease, and autoimmune conditions (17-20). It has also been linked to a wide range of cancers including colorectal cancer, head and neck cancer, prostate cancer, esophageal cancer, and pancreatic cancer (21-24). Studies have shown that markers of systemic inflammation such as C-reactive protein and neutrophil-to-lymphocyte ratio (NLR) can predict outcomes, with high levels associated with poor outcomes (24-26). The exact mechanism is unknown, but inflammation is thought to promote tumor angiogenesis, epithelial to mesenchymal transition, and suppression of cytotoxic T lymphocytes (26). The NLR is a particularly attractive metric since it can be readily calculated from routine blood cell counts.

The prognostic value of NLR in pancreatic cancer treated with radiation therapy has been investigated in only a handful of reports (27-31). Current studies are limited by heterogeneity in clinical outcomes, radiation dose/ fractionation, and time point of NLR (i.e., pre-radiation or post-radiation). Additionally, only two of these studies report on the role of NLR in pancreatic cancer patients treated with stereotactic body radiation therapy (SBRT), with conflicting findings $(27,30)$. As SBRT continues to be explored in pancreatic cancer, an understanding of NLR dynamics and its association with survival outcomes may yield insight into potential immunologic mechanisms of radiation effect, which may have implications on both optimal radiation delivery as well as potential opportunities for combination strategies with immunotherapeutic agents. As such, we report on the association of pre-SBRT and post-SBRT NLR with survival outcomes in a cohort of patients with BRPC/LAPC treated with multi-agent induction chemotherapy followed by five-fraction SBRT. We present the study in accordance with the STROBE reporting checklist (available at https://jgo.amegroups.com/ article/view/10.21037/jgo-21-513/rc).

\section{Methods}

\section{Study design}

This was a single institution review of patients with BRPC or LAPC treated with multi-agent induction chemotherapy followed by five-fraction SBRT from August 2016 to January 2019. The study was conducted in accordance with the Declaration of Helsinki (as revised in 2013), and was approved by the institutional review board of Johns Hopkins University (No.: IRB00285919). Informed consent 
was not taken for this study because it was retrospective in nature and no human experimentation/involvement was taken place.

Inclusion criteria for the study were as follows: (I) biopsy confirmed diagnosis of pancreatic cancer; (II) BRPC or LAPC per NCCN guidelines (2); (III) treatment with multi-agent induction chemotherapy followed by fivefraction SBRT; (IV) complete blood count values before and/or after SBRT available for review.

\section{Treatment details}

Patients were treated with multi-agent induction chemotherapy consisting of FOLFIRINOX (FFX), gemcitabine/nab-paclitaxel (GnP), FFX and GnP, or other regimens. Duration of upfront chemotherapy was at the discretion of the treating medical oncologist but was primarily based on response on interval computed tomography (CT) scans as well as tolerability. Following completion of chemotherapy, patients without distant progression were recommended for five-fraction SBRT. Prior to simulation, patients underwent endoscopic ultrasound-guided fiducial placement to assist with daily setup using cone beam CT (CBCT). At time of simulation, patients were positioned supine with their arms above their head in a Vac-Lok device (CIVCO Medical Solutions, Coralville, IA, USA) for immobilization. Thin-sliced CT scans with intravenous contrast were obtained for treatment planning. Respiratory motion was managed with active breathing control (ABC, Elekta, Stockholm, Sweden). In patients who could not tolerate $\mathrm{ABC}$, a free breathing 4-dimensional (4D)-CT scan was acquired to account for respiratory motion, with an internal target volume (ITV) generated from peak inspiratory and expiratory phases. Target volumes and organs at risk were contoured using Pinnacle Treatment Planning Software (Phillips Radiation Oncology Systems, Fitchburg, WI, USA). The clinical target volume (CTV) consisted of gross disease seen on imaging and the full circumference of involved vasculature. A planning target volume (PTV) was generated by adding a 2-5 $\mathrm{mm}$ isotropic expansion to the CTV in breath-hold cases and to the ITV in free-breathing cases. Pre-treatment and intrafraction CBCT scans were acquired to verify patient positioning. Patients were aligned to bone and then shifted to align to fiducials. All patients were treated on an Elekta linear accelerator unit (Elekta, Stockholm, Sweden). Restaging CT scans were obtained approximately 4 weeks after completion of SBRT. Patients without distant progression and with local vascular involvement that was potentially amenable to complete surgical resection were considered for surgical exploration. Administration of adjuvant chemotherapy was at the discretion of the treating medical oncologist.

\section{Laboratory values}

Laboratory values included hematocrit, platelets, absolute neutrophil count (ANC), and absolute lymphocyte count (ALC). The NLR was obtained by dividing ANC by ALC. The platelet-to-lymphocyte ratio (PLR) was obtained by dividing platelet count by ALC. Laboratory values were collected within 4 weeks prior to the start of SBRT and 1-6 weeks after completion of SBRT. If multiples values existed, the value closest to start of SBRT and closest to 4 weeks after completion of SBRT was recorded.

\section{Statistical analysis}

Patient, disease, and treatment characteristics including age, sex, Karnofsky Performance Status, disease extent, chemotherapy duration/regimen, SBRT dose/fractionation, resection status, and laboratory values were recorded. Differences in median pre-/post-SBRT laboratory values were determined by Mann-Whitney U test. Receiver operating characteristic (ROC) curves were generated to identify the optimal pre-SBRT NLR, post-SBRT NLR, and change in $(\Delta)$ NLR cutoff values using the Youden index. These NLR cutoff values and median values for all other continuous variables were used in statistical analysis. Univariate Cox regression was performed to identify variables associated with OS, LPFS, distant metastasisfree survival (DMFS), and progression-free survival (PFS) from time of SBRT. OS was defined as time from SBRT to death. LPFS and DMFS were defined as time from SBRT to radiographic evidence of local progression and distant progression, respectively. PFS was defined as time from SBRT to radiographic evidence of any progression or death. Variables significant $(\mathrm{P}<0.05)$ on univariate Cox regression were included in multivariable Cox regression. KaplanMeier curves were generated for time to event outcomes, and statistical significance was determined by the log-rank test. A P value $<0.05$ was considered significant throughout the study, with all $\mathrm{P}$ values being 2 -sided. Statistical analyses were performed with JMP version 14.0 (SAS institute, Cary NC, USA) and SPSS version 25.0 (IBM Corporation, Armonk NY, USA). 


\section{Results}

\section{Patient, disease, and treatment characteristics}

Patient, disease, and treatment characteristics are shown in Table 1. From August 2016 to January 2019, 156 patients were treated with multi-agent induction chemotherapy followed by five-fraction SBRT. Median age was 66.4 years (range, $41.7-84.1$ years), and $52 \%$ of patients were male. Borderline resectable disease and LAPC were seen in $41 \%(64 / 156)$ and $59 \%(92 / 156)$ of patients, respectively. The median baseline CA 19-9 was $216.8 \mathrm{U} / \mathrm{mL}$ (range, $<1.0-7,358.4 \mathrm{U} / \mathrm{mL}$ ), and median duration of upfront chemotherapy was 4 months (range, 1-18 months). Chemotherapy regimens consisted of FFX (94/156, 60.2\%), GnP (32/156, 21\%), FFX plus GnP (20/156, 13\%), FFX plus other $(3 / 156,2 \%), \mathrm{GnP}$ plus other $(5 / 156,3 \%)$, and other $(2 / 156,1 \%)$. All patients were treated with fivefraction SBRT. The most common dose/fractionation was 33 Gy/5 fractions (150/156, 96\%), followed by $30 \mathrm{~Gy} / 5$ fractions $(3 / 156,2 \%), 36 \mathrm{~Gy} / 5$ fractions $(2 / 156,1 \%)$, and 30.5 Gy/5 fractions (1/156, 1\%). The majority (106/156, $67 \%)$ of patients underwent surgical resection with Whipple procedure (69/106, 65\%), distal pancreatectomy (34/106, 32 $\%)$, or total pancreatectomy (3/106, 3\%). Post-SBRT/surgery chemotherapy was administered to 58 patients (37.2\%) for a median duration of 2 months (range, 1-6 months).

\section{Laboratory values}

Pre- and post-SBRT laboratory values are displayed in Table 1. Not all laboratory values were available for each patient. Missing values included pre-SBRT ALC (7/156, $4 \%)$, pre-SBRT ANC (7/156, 4\%), pre-SBRT hematocrit (7/156, 4\%), pre-SBRT platelets (7/156, 4\%), post-SBRT ALC (40/156, 26\%), post-SBRT ANC (40/156, 26\%), postSBRT hematocrit $(22 / 156,14 \%)$, and post-SBRT platelets $(22 / 156,14 \%)$. The median pre-SBRT and post-SBRT hematocrit values were $33.3 \%$ (range, 19.9-40.8\%) and $35.9 \%$ (range, $22.5-46.8 \%$ ), respectively. The median preSBRT and post-SBRT platelet counts were $157.0 \times 1,000 / \mu \mathrm{L}$ (range, $40.0-457.0 \times 1,000 / \mu \mathrm{L}$ ) and $153.5 \times 1,000 / \mu \mathrm{L}$ (range, $42.4-416 \times 1,000 / \mu \mathrm{L})$, respectively. The median pre-SBRT and post-SBRT PLR were 108.8 (range, 17.2-1,269.4) and 173.0 (range, 33.9-944.4), respectively. The median pre-SBRT ALC, ANC, and NLR were 1,350 No./ $\mu \mathrm{L}$

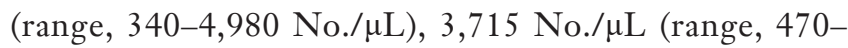
$58,490$ No./ $\mu \mathrm{L})$, and $2.6(0.4-24.1)$, respectively. The median post-SBRT ALC, ANC, and NLR were 840 No./ $\mu \mathrm{L}$

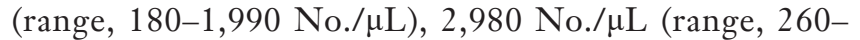
$15,500 \mathrm{No} . / \mu \mathrm{L})$, and $3.3(0.5-42.8)$, with a change of $-37.8 \%$ $(\mathrm{P}<0.001),-19.8 \%(\mathrm{P}=0.014)$, and $+26.9 \%(\mathrm{P}=0.032)$, respectively (Figure 1).

\section{Identification of NLR cutoff values}

From ROC curves and Youden index, the optimal preSBRT NLR, post-SBRT NLR, and $\Delta$ NLR cutoff values in predicting OS were 2.3 (area under the curve: 0.614, sensitivity: $68.8 \%$, specificity: $53.6 \%$ ), 2.6 (area under the curve: 0.598 , sensitivity: $84.4 \%$, specificity: $43.1 \%$ ), and -0.59 (area under the curve: 0.508 , sensitivity: $82.0 \%$, specificity: $34.4 \%$ ), respectively (Figure $2 A-2 C$ ).

\section{Clinical outcomes}

Median follow-up time after SBRT for the entire cohort was 15.1 months (range, $0.3-42.4$ months). At time of last follow-up, $85 / 156$ patients (55\%) had died. Of the patients who were alive, median follow-up time after SBRT was 20.1 months (range, 0.3-42.4 months). The median OS after SBRT was 17.6 months (range, 0.3-42.4 months), with 1-year, 2-year, and 3-year OS rates of $70.5 \%, 45.9 \%$, and $26.7 \%$, respectively. On univariate analysis (UVA), duration of induction chemotherapy, disease grade, resection status, pre-SBRT ANC (threshold 3.7 No./ $\mu$ L), pre-SBRT NLR (threshold 2.3), and post-SBRT NLR (threshold 2.6) were associated with OS (Table 2). Patients with post-SBRT NLR $\geq 2.6$ had a median OS of 16.7 months versus median OS not yet reached in patients with post-SBRT $<2.6(\mathrm{P}=0.009)$ (Figure 3A). On MVA, poorly differentiated disease (HR $=1.82,95 \% \mathrm{CI}: 1.04-3.18, \mathrm{P}=0.035)$, inability to undergo resection ( $\mathrm{HR}=2.17,95 \% \mathrm{CI}: 1.25-3.70, \mathrm{P}=0.006)$, and post-SBRT NLR $\geq 2.6$ (HR $=2.55,95 \%$ CI: $1.20-5.45$, $\mathrm{P}=0.015)$ were associated with inferior OS.

Given that post-SBRT NLR cutoff of 2.6 was significantly associated with OS on MVA, we next determined whether this cutoff value could predict LPFS. The median LPFS after SBRT for the entire cohort was 26.8 months, with 1-, 2-, and 3-year LPFS rates of $73.5 \%$, $51.5 \%$, and $48.9 \%$, respectively. On UVA, baseline CA $19-9$ (threshold $216 \mathrm{U} / \mathrm{mL}$ ), pre-SBRT platelet level (threshold $157 \times 1,000 / \mu \mathrm{L}$ ), and post-SBRT NLR (threshold 2.6) were associated with LPFS (Table 3). Patients with post-SBRT NLR $\geq 2.6$ had a median LPFS of 18.3 months versus median LPFS not yet reached in patients with post-SBRT NLR $<2.6(\mathrm{P}=0.028)$ (Figure 3B). On MVA, only post-SBRT 
Table 1 Patient, treatment, and disease characteristics

\begin{tabular}{|c|c|}
\hline Characteristics & $\begin{array}{c}\mathrm{N}(\%) \text { or median } \\
\text { [range] }\end{array}$ \\
\hline No. of patients & 156 \\
\hline Age (years) & $66.4[41.7-84.1]$ \\
\hline \multicolumn{2}{|l|}{ Sex } \\
\hline Male & $81(51.9)$ \\
\hline Female & $75(48.1)$ \\
\hline \multicolumn{2}{|l|}{ KPS } \\
\hline $90-100$ & $116(74.4)$ \\
\hline $70-80$ & $40(25.6)$ \\
\hline \multicolumn{2}{|l|}{ Histology } \\
\hline Adenocarcinoma & $154(98.8)$ \\
\hline Acinar cell & $1(0.6)$ \\
\hline Undifferentiated carcinoma & $1(0.6)$ \\
\hline \multicolumn{2}{|l|}{ Location of primary tumor } \\
\hline Head & $90(57.7)$ \\
\hline Other & $66(42.3)$ \\
\hline \multicolumn{2}{|l|}{ Disease extent } \\
\hline Borderline resectable & $64(41.0)$ \\
\hline Locally advanced & $92(59.0)$ \\
\hline Baseline CA 19-9 (U/mL) & $216.8[<1.0-7,358.4]$ \\
\hline Induction chemotherapy duration (months) & $4[1-18]$ \\
\hline \multicolumn{2}{|l|}{ Induction chemotherapy } \\
\hline FFX & $94(60.2)$ \\
\hline $\mathrm{GnP}$ & $32(20.5)$ \\
\hline FFX and GnP & $20(12.8)$ \\
\hline FFX plus other & $3(1.9)$ \\
\hline GnP plus other & $5(3.2)$ \\
\hline Other & $2(1.3)$ \\
\hline \multicolumn{2}{|l|}{ SBRT dose and fractionation } \\
\hline 33 Gy in 5 fractions & $150(96.2)$ \\
\hline 30 Gy 5 fractions & $3(1.9)$ \\
\hline 36 Gy in 5 fractions & $2(1.3)$ \\
\hline 30.5 Gy in 5 fractions & $1(0.6)$ \\
\hline PTV $\left(\mathrm{cm}^{3}\right)$ & $87.3[13.1-381.6]$ \\
\hline
\end{tabular}

Table 1 (continued)
Table 1 (continued)

\begin{tabular}{|c|c|}
\hline Characteristics & $\begin{array}{l}\mathrm{N}(\%) \text { or median } \\
\text { [range] }\end{array}$ \\
\hline Surgically resected & $106(67.1)$ \\
\hline Whipple & $69(65.1)$ \\
\hline Distal & $34(32.1)$ \\
\hline Total pancreatectomy & $3(2.8)$ \\
\hline \multicolumn{2}{|l|}{ Post-SBRT/surgery chemotherapy } \\
\hline Yes & $58(37.2)$ \\
\hline No & $91(58.3)$ \\
\hline Unknown & $7(4.5)$ \\
\hline $\begin{array}{l}\text { Post-SBRT/surgery chemotherapy duration } \\
\text { (months) }\end{array}$ & $2[1-6]$ \\
\hline \multicolumn{2}{|l|}{ Pre-SBRT counts } \\
\hline Hct (\%) & $33.3[19.9-40.8]$ \\
\hline Platelets $(\times 1,000 / \mu \mathrm{L})$ & 157.0 [40.0-457.0] \\
\hline 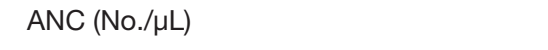 & $3,715[470-58,490]$ \\
\hline 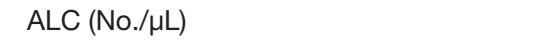 & $1,350[340-4,980]$ \\
\hline NLR & $2.6[0.4-24.1]$ \\
\hline PLR & $108.8[17.2-1,269.4]$ \\
\hline \multicolumn{2}{|l|}{ Post-SBRT counts } \\
\hline Hct (\%) & 35.9 [22.5-46.8] \\
\hline Platelets $(\times 1,000 / \mu \mathrm{L})$ & $153.5[42.4-416]$ \\
\hline 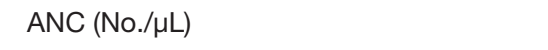 & $2,980[260-15,500]$ \\
\hline 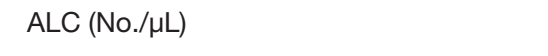 & $840[180-1,990]$ \\
\hline NLR & $3.3[0.5-42.8]$ \\
\hline PLR & 173.0 [33.9-944.4] \\
\hline
\end{tabular}

KPS, Karnofsky Performance Status; CA 19-9, carbohydrate antigen 19-9; FFX, FOLFIRINOX; GnP, gemcitabine/nabpaclitaxel; SBRT, stereotactic body radiation therapy; PTV, planning target volume; Hct, hematocrit; ANC, absolute neutrophil count; ALC, absolute lymphocyte count; NLR, neutrophil-to-lymphocyte ratio; PLR, platelet-to-lymphocyte ratio.

NLR $\geq 2.6$ was associated with inferior LPFS (HR =3.22, 95\% CI: $1.04-9.98, \mathrm{P}=0.043$ ) (Table 3). In the post-SBRT NLR $\geq 2.6$ group, 38\% (31/82) developed local progression compared to $19 \%(6 / 32)$ in the post-SBRT NLR $<2.6$ group $(\mathrm{P}=0.044)$ (Table 4$)$. 
A

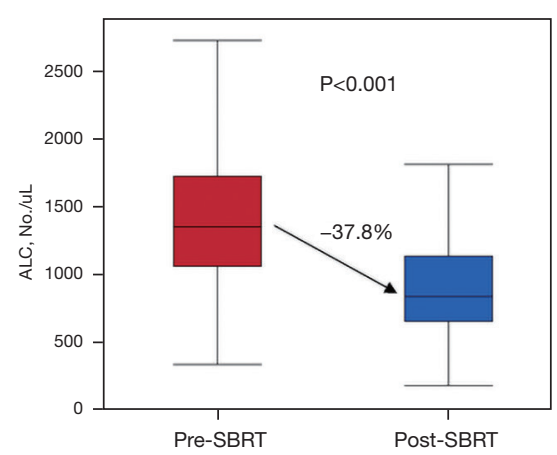

\section{B}

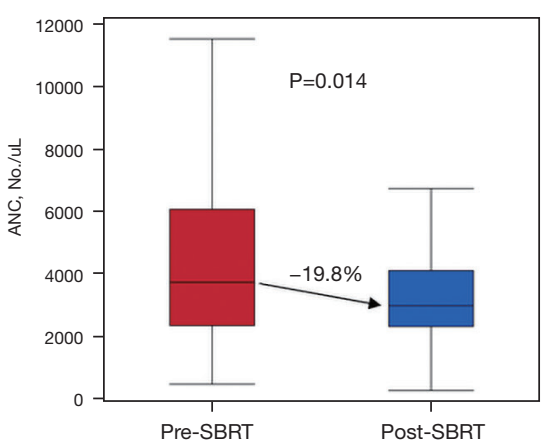

C

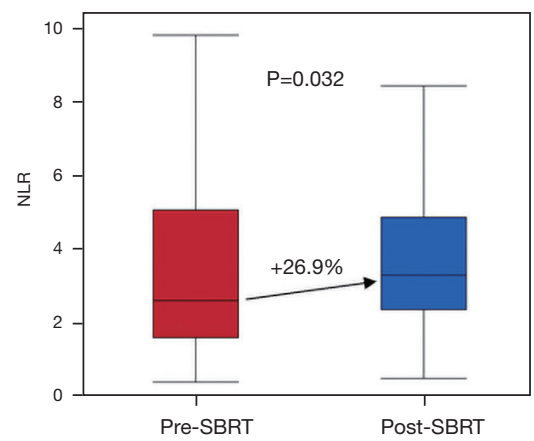

Figure 1 Box plots showing pre- and post-SBRT (A) absolute lymphocyte counts, (B) absolute neutrophil counts, and (C) neutrophil-tolymphocyte ratio. SBRT, stereotactic body radiation therapy.
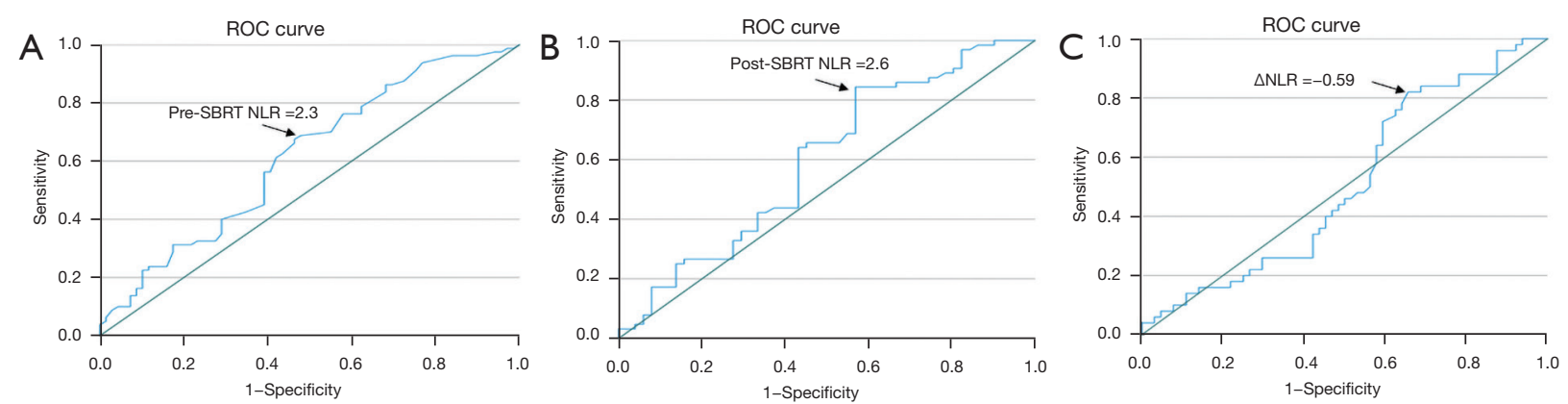

Figure 2 Receiver operating characteristics curves showing the optimal (A) pre-SBRT NLR cutoff value, (B) post-SBRT NLR cutoff value, and (C) change in NLR cutoff value in predicting overall survival. SBRT, stereotactic body radiation therapy; NLR, neutrophil-tolymphocyte ratio.

Tables S1 and S2 show UVA and MVA for DMFS and PFS. On MVA, only poorly differentiated disease was associated worse DMFS (median DMFS: 7.5 vs. 11.5 months, $\mathrm{HR}=1.73,95 \% \mathrm{CI}: 1.09-2.75, \mathrm{P}=0.019$ ). On MVA, poorly differentiated disease (median PFS: 7.5 vs. 10.1 months, $\mathrm{HR}=1.65,95 \% \mathrm{CI}: 1.04-2.62, \mathrm{P}=0.032$ ) and post-SBRT hematocrit $<35.9 \%$ (median PFS: $8.1 \mathrm{vs}$. 11.6 months, HR $=0.52$, 95\% CI: $0.33-0.81, \mathrm{P}=0.004$ ) were associated with worse PFS.

\section{Discussion}

To our knowledge, this is the first study to report on the prognostic value of both pre- and post-SBRT NLR in a cohort of BRPC/LAPC patients treated with multi-agent induction chemotherapy and SBRT. We demonstrate that post-SBRT NLR is strongly associated with clinical outcomes. Specifically, post-SBRT NLR $\geq 2.6$ predicted for worse OS and LPFS in this cohort.

The NLR has been identified as a prognostic factor in a wide range of malignancies, with high levels associated with poor outcomes (21-26). The exact mechanism is unknown. However, studies suggest that neutrophils have protumorigenic effects through secretion of reactive oxygen species, which promote mutagenesis and chemokines/ cytokines, which subsequently promote angiogenesis and tumor proliferation while suppressing cytotoxic $\mathrm{T}$ lymphocytes (32-34). In fact, a recent study of patients enrolled on LAP07, an international phase 3 trial that examined the role of consolidative chemoradiation after upfront chemotherapy in patients with LAPC, showed that baseline and pre-chemoradiation neutrophilia was associated with poor OS and decreased local control (35). Cytotoxic T lymphocytes, on the other hand, have anti-tumorigenic effects (36). A prior report from our institution demonstrated inferior OS in unresected LAPC 
Table 2 Univariate and multivariable analyses of overall survival

\begin{tabular}{|c|c|c|c|c|c|c|}
\hline Variables & \multicolumn{3}{|c|}{ UVA } & \multicolumn{3}{|c|}{ MVA } \\
\hline Age ( $\geq 66$ vs. $<66$ years) & 1.31 & $0.85-2.02$ & 0.214 & & & \\
\hline Sex (male vs. female) & 0.81 & $0.53-1.24$ & 0.332 & & & \\
\hline KPS (> 90 vs. $\leq 90)$ & 0.68 & $0.42-1.12$ & 0.128 & & & \\
\hline Tumor location (head vs. other) & 1.09 & $0.70-1.68$ & 0.715 & & & \\
\hline Induction CT duration ( $\geq 4$ vs. $<4$ months) & 0.45 & $0.27-0.73$ & 0.001 & 0.62 & $0.30-1.31$ & 0.211 \\
\hline Induction CT (FFX vs. GnP) & 0.76 & $0.45-1.30$ & 0.323 & & & \\
\hline Grade (III vs. I/II) & 1.85 & $1.17-2.91$ & 0.008 & 1.82 & $1.04-3.18$ & 0.035 \\
\hline Baseline CA 19-9 ( $\geq 216$ vs. $<216 \mathrm{U} / \mathrm{mL}$ ) & 1.19 & $0.69-2.05$ & 0.524 & & & \\
\hline Pre-SBRT CA $19-9$ ( $\geq 47$ vs. $<47 \mathrm{U} / \mathrm{mL}$ ) & 1.23 & $0.79-1.91$ & 0.368 & & & \\
\hline Post-SBRT CA $19-9$ ( $\geq 47$ vs. $<47 \mathrm{U} / \mathrm{mL})$ & 1.69 & $0.83-3.46$ & 0.151 & & & \\
\hline$\triangle \mathrm{NLR}(\geq-0.59$ vs. $<-0.59)$ & 0.72 & $0.43-1.21$ & 0.220 & & & \\
\hline \multicolumn{7}{|l|}{ Pre-SBRT counts } \\
\hline Hct $(\geq 33.3 \%$ vs. $<33.3 \%)$ & 0.85 & $0.55-1.32$ & 0.463 & & & \\
\hline Platelets ( $\geq 157$ vs. $<157 \times 1,000 / \mu \mathrm{L})$ & 0.83 & $0.44-1.58$ & 0.570 & & & \\
\hline ANC ( $\geq 3.7$ vs. $<3.7$ No. $/ \mu \mathrm{L})$ & 1.59 & $1.02-2.47$ & 0.041 & 1.66 & $0.86-3.22$ & 0.133 \\
\hline Platelets ( $\geq 153.5$ vs. $<153.5 \times 1,000 / \mu \mathrm{L})$ & 0.74 & $0.37-1.46$ & 0.385 & & & \\
\hline ANC ( $\geq 3.0$ vs. $<3.0$ No. $/ \mu \mathrm{L})$ & 1.02 & $0.62-1.66$ & 0.950 & & & \\
\hline ALC ( $\geq 0.8$ vs. $<0.8$ No. $/ \mu \mathrm{L})$ & 0.87 & $0.53-1.45$ & 0.604 & & & \\
\hline $\operatorname{NLR}(\geq 2.6$ vs. $<2.6)$ & 2.39 & $1.21-4.70$ & 0.012 & 2.55 & $1.20-5.45$ & 0.015 \\
\hline $\operatorname{PLR}(\geq 173.0$ vs. $<173.0)$ & 1.09 & $0.67-1.78$ & 0.731 & & & \\
\hline
\end{tabular}

KPS, Karnofsky Performance Status; CA 19-9, carbohydrate antigen 19-9; BRPC, borderline resectable pancreatic cancer; LAPC, locally advanced pancreatic cancer; CT, chemotherapy; FFX, FOLFIRINOX; GnP; gemcitabine/nab-paclitaxel; $\triangle$, change; SBRT, stereotactic body radiation therapy; PTV, planning target volume; Hct, hematocrit; ANC, absolute neutrophil count; ALC, absolute lymphocyte count; NLR, neutrophil-to-lymphocyte ratio; PLR, platelet-to-lymphocyte ratio. 

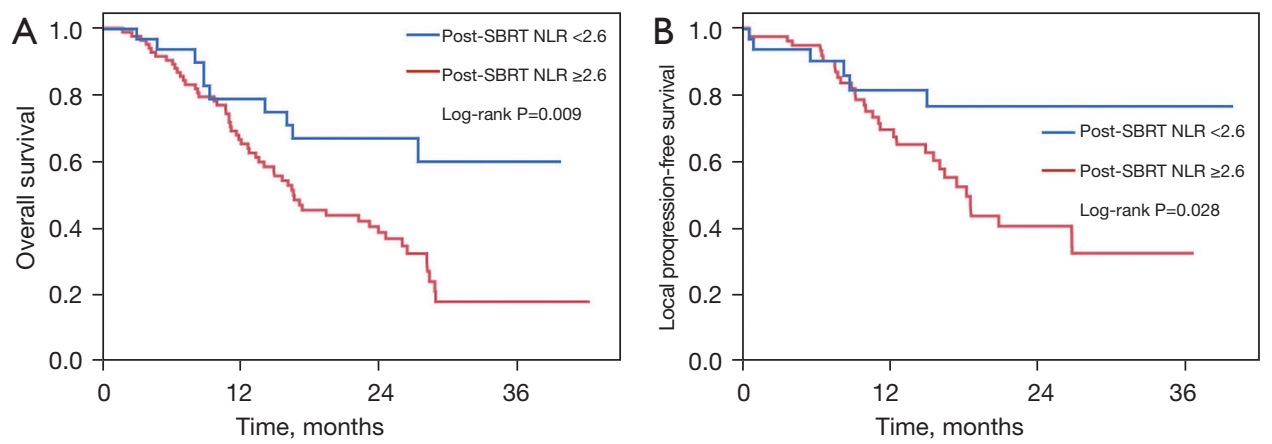

Figure 3 Kaplan-Meier survival curves of (A) overall survival and (B) local progression-free survival stratified by post-SBRT NLR cutoff of 2.6. SBRT, stereotactic body radiation therapy; NLR, neutrophil-to-lymphocyte ratio.

patients who developed grade 3 lymphopenia following chemoradiation (37). These studies suggest that NLR, which takes into account both neutrophils and lymphocytes, may serve as a prognostic measure in pancreatic cancer treated with radiation therapy.

However, until now, few studies have investigated the prognostic role of post-radiation NLR, as opposed to preradiation NLR, given that the former may serve as a better measure of immunologic impact of radiation therapy for pancreatic cancer (27-31). A study by Pearson et al., published in abstract form, represents the only prior report on post-radiation NLR and found that post-SBRT NLR, and not pre-SBRT NLR, was predictive of OS (30). Our data corroborates these findings, with only post-SBRT NLR ( $\geq 2.6)$, and not pre-SBRT NLR, being predictive of outcomes in our cohort. Furthermore, while two prior studies have reported on the association of pre-treatment NLR with local response to therapy, our results are the first to suggest that post-radiation NLR is strongly associated with local control outcomes $(29,31)$.

Our findings also suggest that classic radiobiological mechanisms of DNA damage may not fully encompass radiation effect in this setting and that exploration of radiation-induced immunologic mechanisms represents a key area of continued study (32). Radiation therapy can induce immunogenic death of cancerous cells but can also deplete intratumoral cytotoxic lymphocytes and induce formation of pro-tumorigenic neutrophil extracellular traps $(32,34,38)$. This suggests that there is a fine balance between the anti- and pro-tumorigenic properties of radiation on the tumor microenvironment (TME). As such, further studies are warranted to better understand the complex interplay between radiation therapy and the pancreatic TME, with NLR serving as a potential biomarker of outcomes. Such understanding could have major implications for both optimal radiation design as well opportunities for combination therapy with immunotherapy. As an example, optimal radiation target volume design for pancreatic cancer remains controversial and highly variable, with some data supporting larger volume elective nodal irradiation (39). However, the impact of such volumes on dose to hematopoietic organs and circulating lymphocytes should be considered (40-42). Similarly, conformality and fractionation may also have impact in this regard. Indeed, in a recent study exploring the prognostic value of change in NLR in a cohort of patients with BRPC/LAPC, patients were treated with conventional fractionated radiation (median, $36 \mathrm{~Gy} / 15$ fractions) and experienced a mean increase in NLR of $+99.7 \%$, far greater than the median change in NLR of $+26.9 \%$ experienced by patients in our cohort. Likewise, patients in the aforementioned study by Pearson et al. (30), in which SBRT was administered as well, also experienced a median increase in NLR of only $+27.7 \%$. These findings suggest that radiation technique may significantly impact NLR dynamics. Ultimately, further work to clarify such relationship mechanistically is warranted. Similarly, a better understanding of the manner in which radiation both promotes and disrupts immunologic processes may better inform those combination strategies that merit further study (43-47).

There are several limitations of this study including its single institution retrospective design, limiting generalizability of the findings. Furthermore, laboratory values were collected at varying times, anywhere from 1 day to 4 weeks prior to SBRT and from 1 week to 6 weeks after completion of SBRT. It is possible that these values may have fluctuated during these intervals. Additionally, patients received various upfront systemic therapy regimens 
Table 3 Univariate and multivariable analyses of local progression-free survival

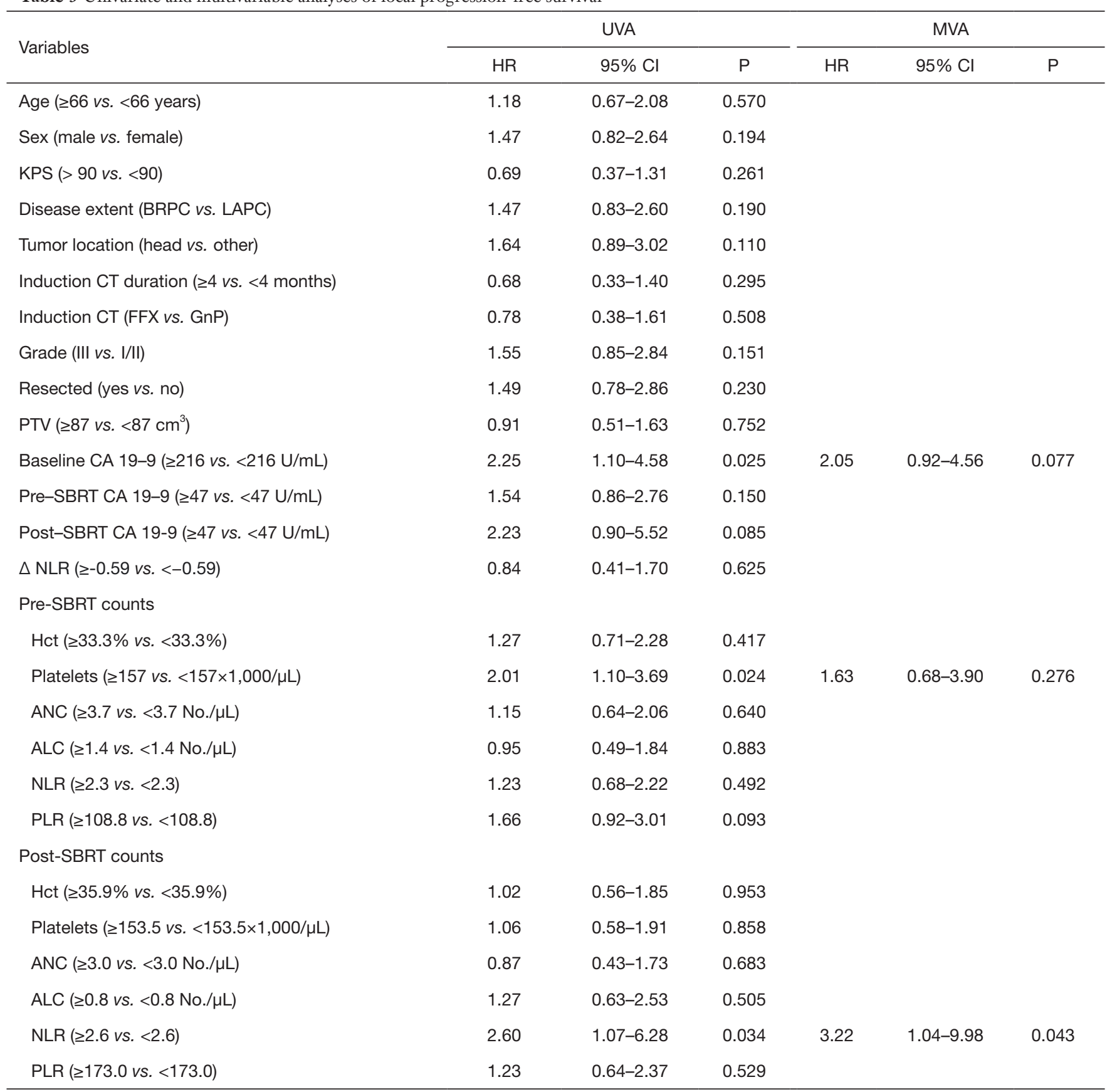

HR, hazard ratio; KPS, Karnofsky Performance Status; CA 19-9, carbohydrate antigen 19-9; BRPC, borderline resectable pancreatic cancer; LAPC, locally advanced pancreatic cancer; CT, chemotherapy; FFX, FOLFIRINOX; GnP; gemcitabine/nab-paclitaxel; $\triangle$, change; SBRT, stereotactic body radiation therapy; PTV, planning target volume; Hct, hematocrit; ANC, absolute neutrophil count; ALC, absolute lymphocyte count; NLR, neutrophil-to-lymphocyte ratio; PLR, platelet-to-lymphocyte ratio. 
Table 4 Development of local progression stratified by post-SBRT NLR cutoff

\begin{tabular}{lccc}
\hline \multirow{2}{*}{ Variables } & No. of patients & \multicolumn{2}{c}{ Local progression, $\mathrm{n}(\%)$} \\
\cline { 3 - 4 } & & Yes & No \\
\hline Post-SBRT NLR $\geq 2.6$ & 82 & $31(37.8)$ & $51(62.2)$ \\
Post-SBRT NLR $<2.6$ & 32 & $6(18.8)$ & $26(81.2)$ \\
\hline
\end{tabular}

SBRT, stereotactic body radiation therapy; NLR, neutrophil-lymphocyte ratio.

including FFX, GnP, or a combination, which in turn, may have influenced laboratory values. Of note, upon subgroup analysis of patients who only received FFX and of patients who only received GnP, post-SBRT NLR still predicted for clinical outcomes. The strengths of this study are its large sample size, homogenous SBRT dose/fractionation regimen (150/156 receiving 33 Gy/5 fractions), and long follow-up time. Despite the limitations, these findings are in agreement with those from other studies and adds novel information about the role of NLR in pancreatic cancer treated with SBRT (27-31).

In conclusion, we demonstrate that post-SBRT NLR (threshold of $\geq 2.6$ ) is associated with clinical outcomes following SBRT, with worse OS and LPFS observed in the high post-SBRT NLR group. These findings highlight the importance of further elucidating the immunologic effects of radiation therapy, which may have implications on radiation design and selection of combination strategies.

\section{Acknowledgments}

Funding: None.

\section{Footnote}

Reporting Checklist: The authors have completed the STROBE reporting checklist. Available at https://jgo. amegroups.com/article/view/10.21037/jgo-21-513/rc

Data Sharing Statement: Available at https://jgo.amegroups. com/article/view/10.21037/jgo-21-513/dss

Conflicts of Interest: All authors have completed the ICMJE uniform disclosure form (available at https://jgo.amegroups. com/article/view/10.21037/jgo-21-513/coif). JMH is a former employee of Pancreatic Action Network and current employee of 1440 foundation. JMH serves as an unpaid editorial board member of fournal of the Gastrointestinal Oncology from January 2021 to December 2022. JM receives royalties from Uptodate and Springer and honoraria from Springer. The other authors have no conflicts of interest to declare.

Ethical Statement: The authors are accountable for all aspects of the work in ensuring that questions related to the accuracy or integrity of any part of the work are appropriately investigated and resolved. The study was conducted in accordance with the Declaration of Helsinki (as revised in 2013). The study was approved by institutional ethics board of Johns Hopkins University (No.: IRB00285919). Informed consent was not taken for this study because it was retrospective in nature and no human experimentation/involvement was taken place.

Open Access Statement: This is an Open Access article distributed in accordance with the Creative Commons Attribution-NonCommercial-NoDerivs 4.0 International License (CC BY-NC-ND 4.0), which permits the noncommercial replication and distribution of the article with the strict proviso that no changes or edits are made and the original work is properly cited (including links to both the formal publication through the relevant DOI and the license). See: https://creativecommons.org/licenses/by-nc-nd/4.0/.

\section{References}

1. Siegel RL, Miller KD, Fuchs HE, et al. Cancer Statistics, 2021. CA Cancer J Clin 2021;71:7-33.

2. National Comprehensive Cancer Network. Pancreatic Adenocarcinoma. Version 2.2021). Available online: https://www.nccn.org/professionals/physician_gls/pdf/ pancreatic_blocks.pdf. Accessed 7/15/2021.

3. Rawla P, Sunkara T, Gaduputi V. Epidemiology of Pancreatic Cancer: Global Trends, Etiology and Risk Factors. World J Oncol 2019;10:10-27.

4. Hammel P, Huguet F, van Laethem JL, et al. Effect of Chemoradiotherapy vs chemotherapy on survival in patients with locally advanced pancreatic cancer 
controlled after 4 months of gemcitabine with or without Erlotinib: the LAP07 randomized clinical trial. JAMA 2016;315:1844-53.

5. Iacobuzio-Donahue CA, Fu B, Yachida S, et al. DPC4 gene status of the primary carcinoma correlates with patterns of failure in patients with pancreatic cancer. J Clin Oncol 2009;27:1806-13.

6. Cardillo N, Seible DM, Fero KE, et al. Clinical Impact of Local Progression in Pancreatic Cancer. J Natl Compr Canc Netw 2018;16:711-7.

7. Sadot E, Doussot A, O'Reilly EM, et al. FOLFIRINOX Induction Therapy for Stage 3 Pancreatic Adenocarcinoma. Ann Surg Oncol 2015;22:3512-21.

8. Sherman WH, Chu K, Chabot J, et al. Neoadjuvant gemcitabine, docetaxel, and capecitabine followed by gemcitabine and capecitabine/radiation therapy and surgery in locally advanced, unresectable pancreatic adenocarcinoma. Cancer 2015;121:673-80.

9. Gemenetzis G, Groot VP, Blair AB, et al. Survival in Locally Advanced Pancreatic Cancer After Neoadjuvant Therapy and Surgical Resection. Ann Surg 2019;270:340-7.

10. Truty MJ, Kendrick ML, Nagorney DM, et al. Factors Predicting Response, Perioperative Outcomes, and Survival Following Total Neoadjuvant Therapy for Borderline/Locally Advanced Pancreatic Cancer. Ann Surg 2021;273:341-9.

11. Mellon EA, Hoffe SE, Springett GM, et al. Long-term outcomes of induction chemotherapy and neoadjuvant stereotactic body radiotherapy for borderline resectable and locally advanced pancreatic adenocarcinoma. Acta Oncol 2015;54:979-85.

12. Ferrone CR, Marchegiani G, Hong TS, et al. Radiological and surgical implications of neoadjuvant treatment with FOLFIRINOX for locally advanced and borderline resectable pancreatic cancer. Ann Surg 2015;261:12-7.

13. Faris JE, Blaszkowsky LS, McDermott S, et al. FOLFIRINOX in locally advanced pancreatic cancer: the Massachusetts General Hospital Cancer Center experience. Oncologist 2013;18:543-8.

14. Jang JY, Han Y, Lee H, et al. Oncological benefits of neoadjuvant chemoradiation with gemcitabine versus upfront surgery in patients with borderline resectable pancreatic cancer: a Prospective, randomized, open-label, multicenter phase 2/3 trial. Ann Surg. 2018;268:215-22.

15. Versteijne E, van Eijck CH, Punt CJ, et al. Preoperative radiochemotherapy versus immediate surgery for resectable and borderline resectable pancreatic cancer (PREOPANC trial): study protocol for a multicentre randomized controlled trial. Trials 2016;17:127.

16. Katz MHG, Shi Q, Meyers JP, et al. Alliance A021501: Preoperative mFOLFIRINOX or mFOLFIRINOX plus hypofractionated radiation therapy (RT) for borderline resectable (BR) adenocarcinoma of the pancreas. J Clin Oncol 2021 39:3_suppl:377.

17. Barnes PJ. Inflammatory mechanisms in patients with chronic obstructive pulmonary disease. J Allergy Clin Immunol 2016;138:16-27.

18. Lopez-Candales A, Hernández Burgos PM, HernandezSuarez DF, et al. Linking Chronic Inflammation with Cardiovascular Disease: From Normal Aging to the Metabolic Syndrome. J Nat Sci 2017;3:e341.

19. Duan L, Rao X, Sigdel KR. Regulation of Inflammation in Autoimmune Disease. J Immunol Res 2019;2019:7403796.

20. Coussens LM, Werb Z. Inflammation and cancer. Nature 2002;420:860-7.

21. Howard R, Kanetsky PA, Egan KM. Exploring the prognostic value of the neutrophil-to-lymphocyte ratio in cancer. Sci Rep 2019;9:19673.

22. Murata M. Inflammation and cancer. Environ Health Prev Med 2018;23:50.

23. Grivennikov SI, Greten FR, Karin M. Immunity, Inflammation, and Cancer. Cell. 2010;140:883-9.

24. Yin Y, Wang J, Wang X, et al. Prognostic value of the neutrophil to lymphocyte ratio in lung cancer: A metaanalysis. Clinics (Sao Paulo) 2015;70:524-30.

25. Kishi T, Nakamura A, Itasaka S, et al. Pretreatment C-reactive protein level predicts outcome and patterns of failure after chemoradiotherapy for locally advanced pancreatic cancer. Pancreatology 2015;15:694-700.

26. Zhou Y, Wei Q, Fan J, et al. Prognostic role of the neutrophil-to-lymphocyte ratio in pancreatic cancer: A meta-analysis containing 8252 patients. Clin Chim Acta 2018;479:181-9.

27. Alagappan M, Pollom EL, von Eyben R, et al. Albumin and Neutrophil-Lymphocyte Ratio (NLR) Predict Survival in Patients With Pancreatic Adenocarcinoma Treated With SBRT. Am J Clin Oncol 2018;41:242-7.

28. Wolfe AR, Siedow M, Nalin A, et al. Increasing neutrophil-to-lymphocyte ratio following radiation is a poor prognostic factor and directly correlates with splenic radiation dose in pancreatic cancer. Radiother Oncol 2021;158:207-14.

29. Hasegawa S, Eguchi H, Tomokuni A, et al. Pre-treatment neutrophil to lymphocyte ratio as a predictive marker for pathological response to preoperative chemoradiotherapy in pancreatic cancer. Oncol Lett 2016;11:1560-6. 
30. Pearson AL, Jin W, Mellon EA, et al. Post-Stereotactic Body Radiation Therapy (SBRT) Neutrophil-toLymphocyte Ratio (NLR) in Patients With Borderline Resectable Pancreatic Cancer (BRPC) May Be a Prognostic Biomarker. Int J Radiat Oncol Biol Phys. 2016; 96:E153.

31. Lee BM, Chung SY, Chang JS, et al. The NeutrophilLymphocyte Ratio and Platelet-Lymphocyte Ratio Are Prognostic Factors in Patients with Locally Advanced Pancreatic Cancer Treated with Chemoradiotherapy. Gut Liver 2018;12:342-52.

32. Jarosz-Biej M, Smolarczyk R, Cichoń T, et al. Tumor Microenvironment as A "Game Changer" in Cancer Radiotherapy. Int J Mol Sci. 2019;20:3212.

33. de Kleijn S, Langereis JD, Leentjens J, et al. IFN$\gamma$-Stimulated Neutrophils Suppress Lymphocyte Proliferation through Expression of PD-L1. PLoS One. 2013;8:e72249.

34. Wu L, Saxena S, Awaji M, et al. Tumor-Associated Neutrophils in Cancer: Going Pro. Cancers (Basel) 2019; 11:564.

35. Schernberg A, Vernerey D, Goldstein D, et al. Predictive Value of Neutrophils Count for Local Tumor Control After Chemoradiotherapy in Patients With Locally Advanced Pancreatic Carcinoma. Int J Radiat Oncol Biol Phys 2021;110:1022-31.

36. Farhood B, Najafi M, Mortezaee K. CD8+ cytotoxic T lymphocytes in cancer immunotherapy: A review. J Cell Physiol 2019;234:8509-21.

37. Wild AT, Ye X, Ellsworth SG, et al. The Association Between Chemoradiation-related Lymphopenia and Clinical Outcomes in Patients With Locally Advanced Pancreatic Adenocarcinoma. Am J Clin Oncol 2015;38:259-65.

38. Shinde-Jadhav S, Mansure JJ, Rayes RF, et al. Role of neutrophil extracellular traps in radiation resistance of invasive bladder cancer. Nat Commun 2021;12:2776.

39. Miller JA, Toesca DAS, Baclay JRM, et al. Pancreatic Stereotactic Body Radiation Therapy with or without Hypofractionated Elective Nodal Irradiation. Int J Radiat Oncol Biol Phys 2022;112:131-42.

40. Deek MP, Benenati B, Kim S, et al. Thoracic Vertebral Body Irradiation Contributes to Acute Hematologic Toxicity During Chemoradiation Therapy for NonSmall Cell Lung Cancer. Int J Radiat Oncol Biol Phys 2016;94:147-54.

41. Chadha AS, Liu G, Chen HC, et al. Does Unintentional Splenic Radiation Predict Outcomes After Pancreatic
Cancer Radiation Therapy? Int J Radiat Oncol Biol Phys 2017;97:323-32.

42. Wild AT, Herman JM, Dholakia AS, et al. LymphocyteSparing Effect of Stereotactic Body Radiation Therapy in Patients With Unresectable Pancreatic Cancer. Int J Radiat Oncol Biol Phys 2016;94:571-9.

43. ClincialTrials.gov [Internet]. Bethesda (MD): National Library of Medicine (US). 2000 Feb 29. Identifier NCT03161379. A Phase II Clinical Trial of GVAX Pancreas Vaccine (With Cyclophosphamide) in Combination With Nivolumab and Stereotactic Body Radiation Therapy (SBRT) Followed by Definitive Resection for Patients With Borderline Resectable Pancreatic Adenocarcinoma. May 19, 2017. Available online: https://clinicaltrials.gov/ct2/show/NCT03161379

44. ClincialTrials.gov [Internet]. Bethesda (MD): National Library of Medicine (US). 2000 Feb 29. Identifier NCT02648282. A Phase II Study of GM-CSF Secreting Allogeneic Pancreatic Cancer Vaccine in Combination With PD-1 Blockade Antibody (Pembrolizumab) and Stereotactic Body Radiation Therapy (SBRT) for the Treatment of Patients With Locally Advanced Adenocarcinoma of the Pancreas. Available online: https:// clinicaltrials.gov/ct2/show/NCT02648282

45. ClincialTrials.gov [Internet]. Bethesda (MD): National Library of Medicine (US). 2000 Feb 29. Identifier NCT03563248. A Randomized Phase 2 Study of Losartan and Nivolumab in Combination With FOLFIRINOX and SBRT in Localized Pancreatic Cancer. Available online: https://clinicaltrials.gov/ct2/show/NCT03563248

46. Bockorny B, Semenisty V, Macarulla T, et al. BL-8040, a CXCR4 antagonist, in combination with pembrolizumab and chemotherapy for pancreatic cancer: the COMBAT trial. Nat Med 2020;26:878-85.

47. Faget J, Peters S, Quantin X, et al. Neutrophils in the era of immune checkpoint blockade. J Immunother Cancer 2021;9:e002242.

Cite this article as: Reddy AV, Hill CS, Sehgal S, He J, Zheng L, Herman JM, Meyer J, Narang AK. High neutrophil-tolymphocyte ratio following stereotactic body radiation therapy is associated with poor clinical outcomes in patients with borderline resectable and locally advanced pancreatic cancer. J Gastrointest Oncol 2022;13(1):368-379. doi: 10.21037/jgo-21-513 


\section{Supplementary}

Table S1 Univariate and multivariable analyses of distant metastasis-free survival

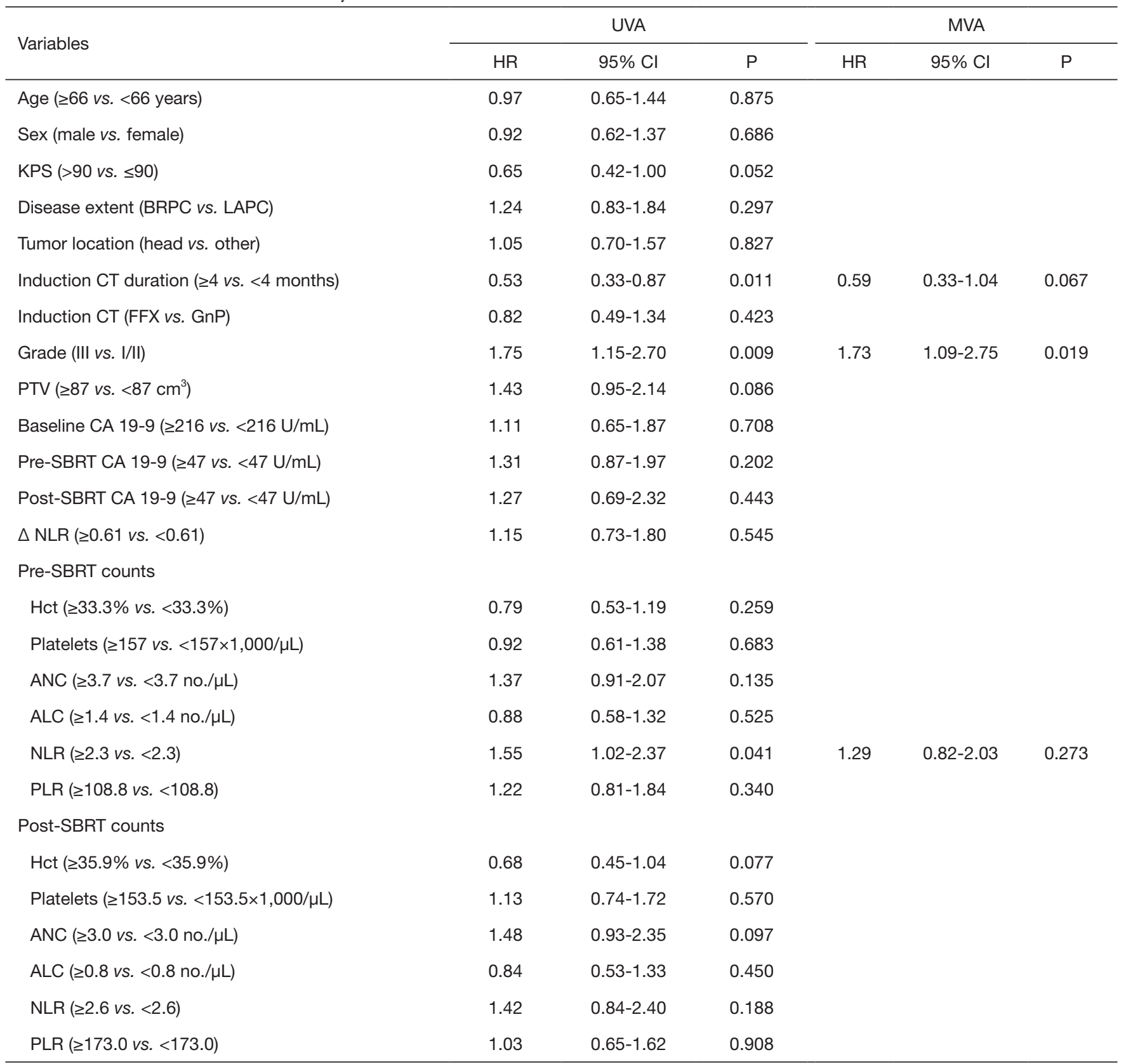

KPS, Karnofsky Performance Status; CA 19-9, carbohydrate antigen 19-9; BRPC, borderline resectable pancreatic cancer; LAPC, locally advanced pancreatic cancer; CT, chemotherapy; FFX, FOLFIRINOX; GnP; gemcitabine/nab-paclitaxel; $\triangle$, change; SBRT, stereotactic body radiation therapy; PTV, planning target volume; Hct, hematocrit; ANC, absolute neutrophil count; ALC, absolute lymphocyte count; NLR, neutrophil-to-lymphocyte ratio; PLR, platelet-to-lymphocyte ratio. 
Table S2 Univariate and multivariable analyses of progression-free survival

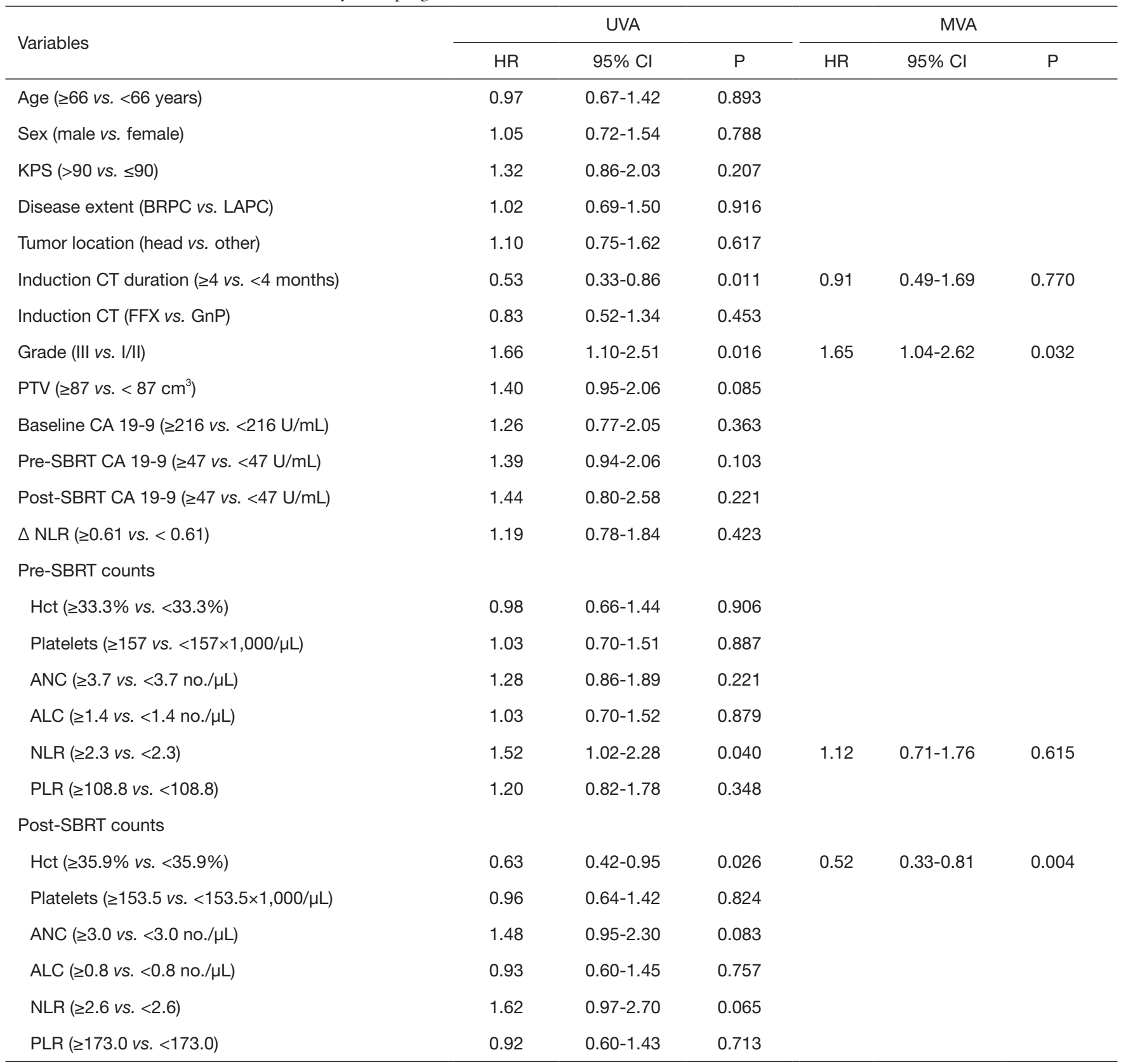

KPS, Karnofsky Performance Status; CA 19-9, carbohydrate antigen 19-9; BRPC, borderline resectable pancreatic cancer; LAPC, locally advanced pancreatic cancer; CT, chemotherapy; FFX, FOLFIRINOX; GnP; gemcitabine/nab-paclitaxel; $\Delta$, change; SBRT, stereotactic body radiation therapy; PTV, planning target volume; Hct, hematocrit; ANC, absolute neutrophil count; ALC, absolute lymphocyte count; NLR, neutrophil-to-lymphocyte ratio; PLR, platelet-to-lymphocyte ratio. 